\title{
ESTADO DE LA PERIURBANIZACION EN FRANCIA: EL CASO DE NANCY
}

\author{
Rafael DE MIGUEL* \\ Departamento de Geografía y Ordenación del Territorio. Universidad de Zaragoza \\ Institut d'Urbanisme de Paris
}

\begin{abstract}
Resumen: Este artículo pretende mostrar el proceso de periurbanización en Nancy (Francia) y las operaciones de ordenación urbana que ha necesitado en su configuración espacial. Tras una descripción del fenómeno en Francia, Nancy es puesta en relación con el resto del sistema urbano francés. Posteriormente, se analizan las categorías morfológicas resultantes de este proceso y las funciones que desempeña este espacio en el conjunto de la aglomeración.
\end{abstract}

Palabras clave: Ordenación periurbana, lotissement, zone d'aménagement concerté, zona de actividad, movimientos pendulares, frente de urbanización.

\begin{abstract}
The aim of this article is showing, how the process of periurbanization has been developed in Nancy and also, the management actions for the territorial shaping. The author describes the general tendences in France, which urban system Nancy is placed in. Finally, morphological and fonctional analyses of Nancy's rural-urban fringe are exposed in conexion with the hole of the urban aglomeration.
\end{abstract}

Key words: Periurban management, lotissement, zone d'aménagement concerté, activity area, daily migrations, urbanisation front.

\footnotetext{
* Este artículo es un resumen del trabajo de investigación realizado durante la estancia en Nancy el curso pasado, gracias al cuadro europeo de programas Erasmus. Los profesores Humbert (Univ. de Nancy) y Bielza de Ory, coordinadores del programa n 93-F-3089/07, han sido al mismo tiempo los codirectores de dicha investigación.
} 


\section{SITUACION ACTUAL DEL FENOMENO DE PERIURBANIZACION EN FRANCIA}

A finales de los años sesenta, se observa en las grandes capitales francesas el fenómeno metropolitano de crecimiento expansivo, materializado espacialmente gracias a la difusión de la vivienda unifamiliar. La edificación del hábitat individual se opone y complementa a los grandes bloques de habitat colectivo, que se habían construído en los años cincuenta y sesenta siguiendo las pautas del urbanismo progresista de Le Corbusier. A partir de esa fecha y hasta la actualidad, se desarrolla la tipología de lotissement o urbanización de un número variable de chalets, desde unas pocas unidades hasta sobrepasar el millar de pavillons.

La novedad geográfica reside, no tanto en el hecho de la aparición de hábitat individual en medio urbano (puesto que ya había conocido una fase de expansión en el período de entreguerras), como el que éste urbanice las áreas rurales periféricas de la aglomeración sin una continuidad espacial con el núcleo central, es decir de manera diferente a como se había edificado hasta el momento. Este fenómeno se desarrolla como si la ciudad se proyectase en el medio rural de una manera rota en fragmentos, despedazada o éparpillée (de ahí el título del libro de Bauer y Roux).

La aplicación de las teorías howardianas de la ciudad jardín se dió con bastante anterioridad en países como Estados Unidos, Gran Bretaña, Alemania, Países Bajos o países escandinavos. El retraso de Francia en relación a estos países se explica a partir de una serie de variables. Hasta que no maduraron en Francia unas condiciones económicas, técnicas, sociales y en especial de planificación e instrumentación urbana, no comenzó la periurbanización.

La elevación del nivel de vida y poder adquisitivo de las familias francesas, así como la oferta de modalidades de financiación de la vivienda a medio y largo plazo, han permitido desde mediados de los años sesenta el acceso a una vivienda de mayor coste. Al mismo tiempo, la aparición de nuevas fórmulas comerciales en la periferia y la aplicación de la tecnología en la conservación de alimentos, en la industrialización de la construcción individual y en lo referente a transportes y comunicaciones, coadyuvan a dinamizar este proceso.

Ello no se entiende sin la democratización del automóvil, puesto que en un primer estadio el residente periurbano mantiene su empleo en el centro de la ciudad, por lo que necesita desplazarse a diario. La modernización de la red viaria y la construcción de numerosos tramos de autopista en medio periurbano son otras claves del complejo, al igual que la incorporación progresiva de la mujer al mundo laboral y la búsqueda de una vivienda mayor. En una época en la que el crecimiento demográfico explosivo de postguerra se ha ralentizado en Francia, lo que crece ahora de manera 
exponencial son los usos del suelo. Ligado a los conceptos de "calidad de vida" y "sociedad del ocio" aparece el anhelo de vivir "en contacto con la naturaleza", con proximidad a espacios verdes, de recreo, etc.

Toda esta serie de condicionantes que caracterizan a la sociedad neoindustrial aparecen interrelacionados entre sí y coinciden cronológicamente en Francia con la creación de la DATAR en 1963 y con la entrada en vigor de la Ley del Suelo en 1967. A partir de ahora se aplica una larga serie de reglamentación urbanística, dentro de la cual destacan las figuras de POS $^{1}$ y de manera casi específica al medio periurbano, la ZAC. ${ }^{2}$.

La síntesis de todo lo descrito anteriormente converge en un crecimiento periurbano inusitado en Francia durante estos últimos veinticinco años; crecimiento del que cabe discernir dos fases: una primera ola de gran demanda de suelo periurbano, por lo tanto de gran crecimiento cuantitativo de usos del suelo y de multiplicación estereotipada de lotissements y Zonas de Actividad, hasta mediados de los años ochenta. Actualmente existe una segunda etapa, complementando al nuevo fenómeno de rehabilitación y reconquista del centro, en la que el crecimiento periurbano no es tanto cuantitativo como cualitativo: el frente de urbanización ralentiza su avance en el medio rural y lo que ahora se potencia es la creación de zonas de actividad de alta tecnología y V.A.B. y de zonas residenciales de mayor calidad y menor tamaño.

Frente a un crecimiento periurbano explosivo en los inicios, las tendencias actuales de ordenación periurbana son las de promover espacios de calidad y las de corregir los desequilibrios territoriales, generalmente mínimos, ya que la correcta aplicación de las ZAC ha permitido que los espacios periurbanos de las metrópolis regionales francesas sean generalmente bastante armónicos.

En origen, el espacio periurbano tiene como primera función la residencia de activos urbanos en el núcleo central. Conforme se va desarrollando un espacio

1 Plan de Ocupación de Suelos. Es la calificación del uso del suelo, de una manera equivalente a los PGOU españoles.

2 Zones d'Aménagement Concerté. Son el gran útil del urbanismo francés en los últimos veinticinco años. Tienen por objeto "la ordenación y equipamiento de terrenos, edificados o no edificados, con vista a la realización de construcciones con una finalidad residencial, comercial, industrial, de servicios o equipamientos públicos y privados". Es un buen ejemplo de concertación y acuerdo entre colectividades públicas y promotores privados. Para llevar a cabo la urbanización de una ZAC es necesario un dossier de realización que comprende: el programa de equipamientos públicos, el presupuesto estimado y el Plan de Ordenación de Zona (reglamento, documentos gráficos, informes técnicos, etc.) 
periurbano, se busca un equilibrio entre residencia y actividad. La expresión territorial de las actividades económicas en el espacio periurbano francés adquiere características específicas, en concreto con las realidades periurbanas de plataformas multimodales, parques tecnológicos y grandes superficies comerciales, los cuales siguen modelos particulares de planificación, gestión y ordenación periurbana, diferenciándose así de otros países occidentales.

\section{INTEGRACION DE NANCY EN EL CONJUNTO DEL ESPACIO PERIURBANO FRANCES}

Cabe distinguir, como consecuencia del proceso de periurbanizacón en Francia, tres tipologías bastante bien diferenciadas: el espacio periurbano en sentido literal, el espacio rururbano y la periurbanización turística.

El espacio periurbano como tal es aquel que se refiere al territorio que rodea a las grandes ciudades, generado en el proceso de desurbanización de un modelo teórico de ciudad concéntrica, a pesar de que la realidad muestra que el espacio periurbano se desarrolla más en aquellas áreas próximas a los nudos de autopistas en las entradas/salidas de las aglomeraciones. En Francia, definitoriamente existe un espacio periurbano como tal en toda ciudad de más de 20.000 habitantes. Buenos ejemplos de ello son las metrópolis regionales, donde el fenómeno periurbano se extiende en un radio de unos veinticinco kilómetros en relación al centro de Nantes, Burdeos, Toulouse, Rennes, Orléans y nuestro caso, Nancy.

Los espacios periurbanos franceses más desarrollados son los de las grandes regiones urbanas o conurbaciones, donde el periurbano de una metrópoli enlaza con el de otra ciudad menor y así sucesivamente; los casos de esta subtipología son el alsaciano (Estrasburgo-Colmar-Mulhouse), la conurbación de Pas de Calais (LilleRoubaix-Tourcoing), el centro de la Depresión del Ródano (Lyon-Villeurbanne-St. Etienne), la región urbana de la Costa Azul (Marsella-Toulon-Cannes-Niza-MonacoMenton) y por supuesto, la Gran Región de París y su prolongación por el bajo Sena, existiendo una continuidad periurbana que se aleja hasta setenta kilómetros del centro de Paris.

El espacio rururbano, llamado tambien la "tercera corona", es el que mantiene un paisaje todavía rural sin grandes impactos ni modificaciones. Si morfológicamente se caracteriza por carecer de una multiplicación de urbanizaciones y por mostrar una edificación bastante discontinua, funcionalmente se define por la ausencia de grandes centros comerciales, de actividad industrial, etc., es decir, por una ocupación mayoritaria en el sector primario. El rasgo que definitivamente acaba por diferenciar 
este espacio rururbano del periurbano, es el tipo de desplazamiento: en el tipo anterior, se trataba de movimientos pendulares o diarios, al existir una disociación espacial entre lugar de residencia y de trabajo. Es el caso de los commuters, mientras que en la tercera corona los desplazamientos son de fin de semana, vacacionales o estivales. En Francia corresponde al gran área de residencias secundarias y de turismo rural de la Borgoña, Seine-et-Marne, Auvergne o el piedemonte del Macizo Central.

Por último, existe una periurbanización masiva, de crecimiento más anárquico, mayor impacto ambiental y mayores desequilibrios verticales, que corresponde a una demanda de suelo con una funcionalidad turística, sea litoral o de montaña. El primero de los casos se manifiesta en el área del Mediodía y la costa atlántica de Aquitania, Bretaña y Normandia. El segundo aparece de manera más difusa a lo largo de las cordilleras de los Vosgos, Alpes y Pirineos.

El espacio periurbano de Nancy se encuentra integrado dentro del grupo de las metrópolis regionales, puesto que la aglomeración nanceienne constituye la cabecera de la jerarquía urbana de la Lorena. El desarrollo de la aglomeración de Nancy ${ }^{3}$ (sistema intraurbano) debe considerarse desde la perspectiva de su rango ${ }^{4}$, tamaño, función y posición ${ }^{5}$ regional (sistema interurbano).

Otro factor que ha incidido positivamente en el crecimiento periurbano de Nancy ha sido su situación dentro de la red urbana francesa. Se encuentra en el cruce de dos ejes de comunicación de importancia europea: el eje este-oeste que conecta, por medio de la Nacional 4 y de la vía del tren, París con Estrasburgo, Stuttgart y Munich; el eje norte-sur materializado en la llamada "autopista del sol", enlaza el Benelux y el Norte de Alemania con otros polos de dinamismo urbano y económico, Lyon y el arco mediterráneo.

El estudio del espacio periurbano de Nancy se refiere a la franja más próxima a la aglomeración, el espacio periurbano en sentido literal, que se extiende hasta unos quince kilómetros del centro de Nancy. Comprende veinticuatro municipios, con un total de 18.807 hectáreas y una población de 183.911 habitantes en 1990.

\footnotetext{
3333.290 habitantes según el censo de1990, frente a 232.258 en 1962, siendo un crecimiento mayoritariamente gracias a los municipios periféricos. En 1950, las viviendas del municipio central de Nancy suponían un $56 \%$ sobre el total de la aglomeración, mientras que en 1990 , solamente un $37 \%$.

${ }^{4}$ Condicionado en parte por su condición de metrópoli de equilibrio después de 1963.

5 En el centro de la pretendida metrópolis regional Thionville-Metz-Nancy-Toul-Luneville.
} 
MORFOLOGIA DEL ESPACIO PERIURBANO DE NANCY. 1968.

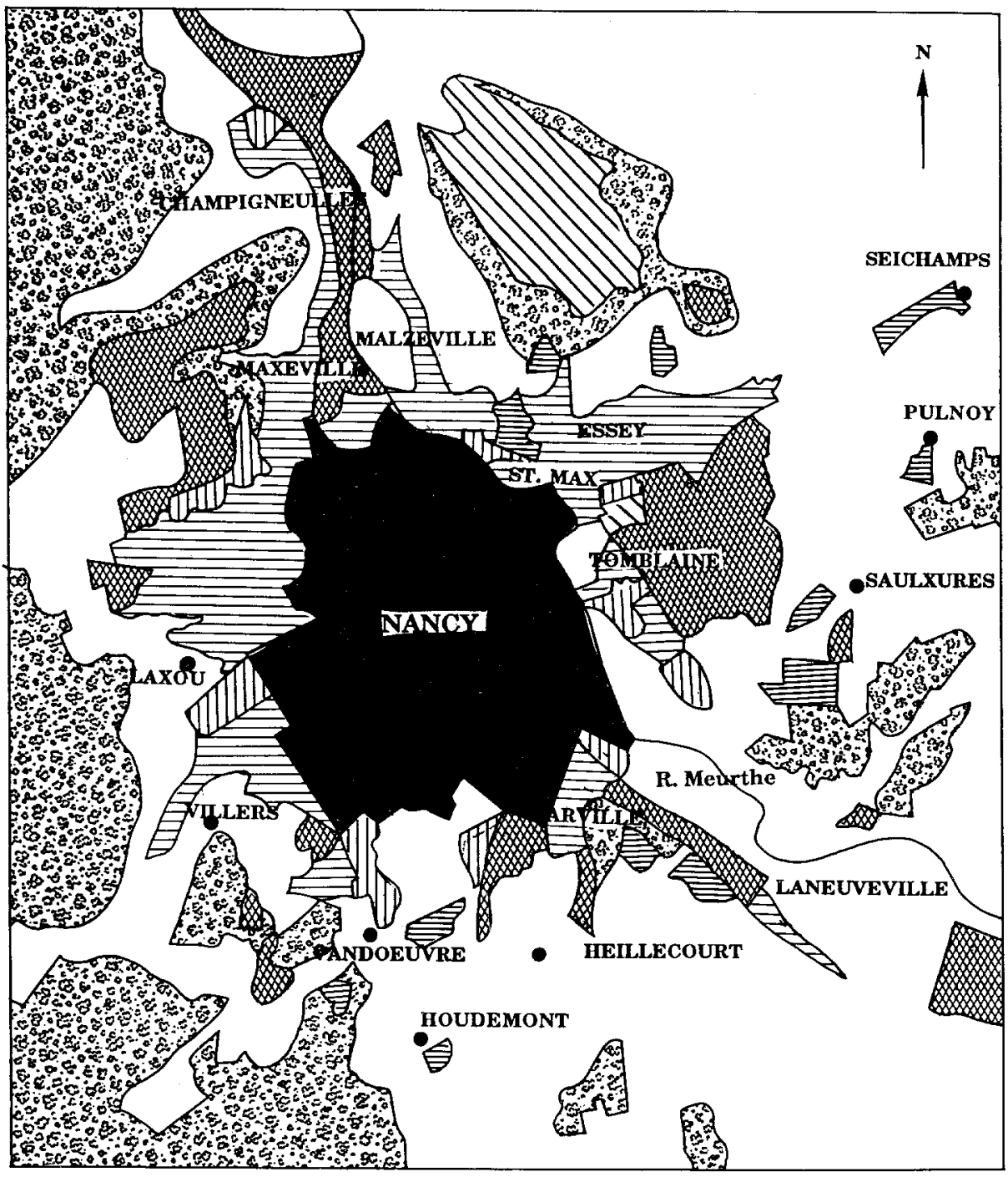

Cartografia elaborada por el autor a partir de fotointerpretación.

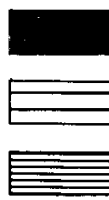

MUNICIPIO CENTRAL

HABITAT INDIVIDUAL ANTIGUO

HABITAT INDIVIDUAL RECIENTE

ППग] HABITAT COLECTIVO a

ZONA DE ACTIVIDAD

MIV zONA DE Ocro

ESPACIOS VERDES

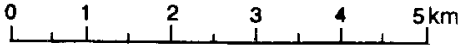


MORFOLOGIA DEL ESPACIO PERIURBANO DE NANCY. 1994.

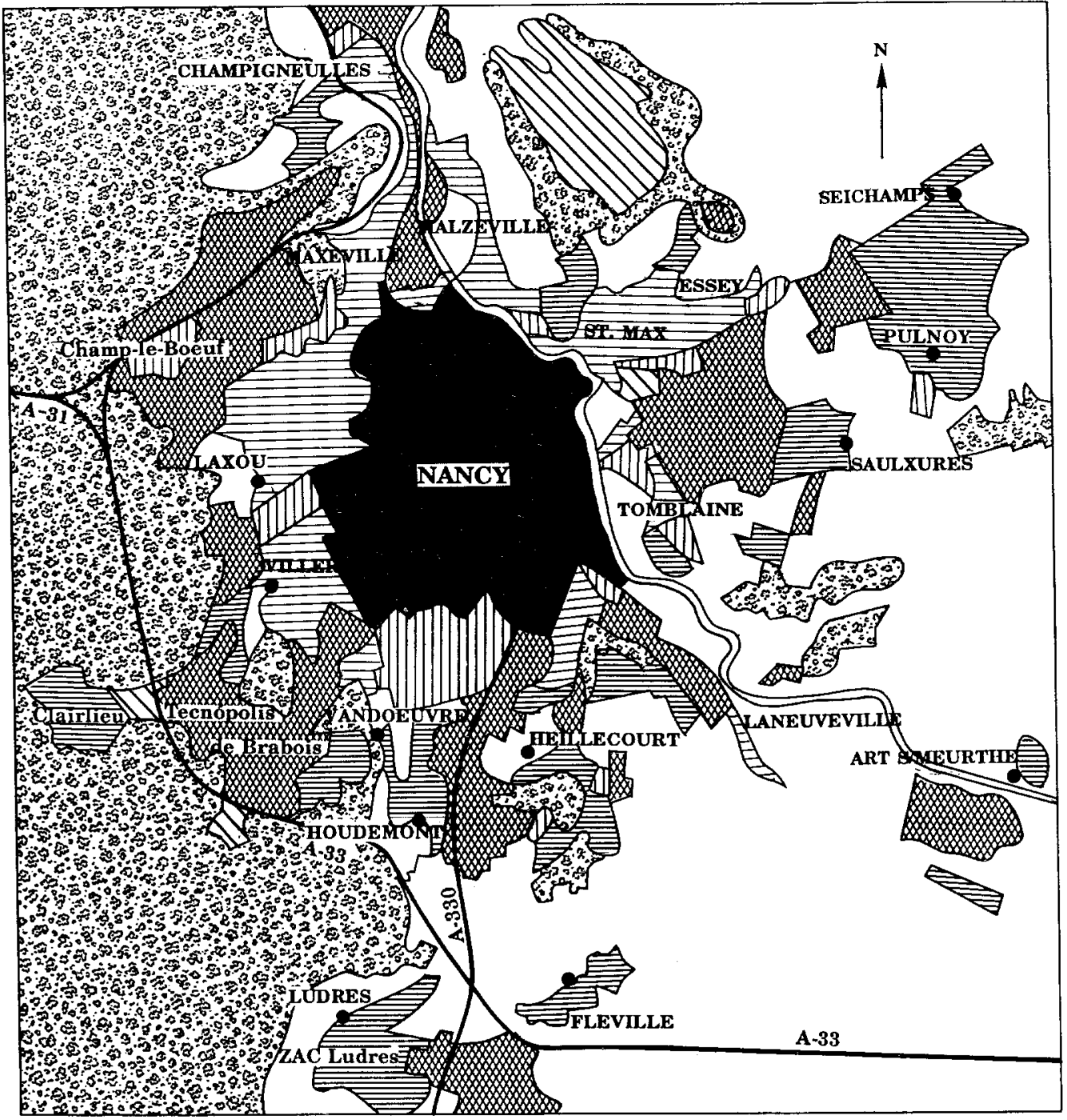

Cartografía elaborada por el autor a partir de fotointerpretación.

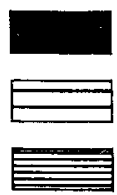

MUNICIPIO CENTRAL

HABITAT INDIVIDUAL ANTIGUO

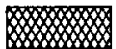

ZONA DE ACTIVIDAD

HABITAT INDIVIDUAL, RECIENTE

DSV zONA DE OCIO

ESP?

HABITAT COLECTIVo

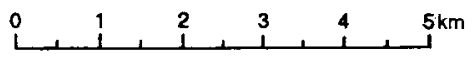




\section{8-1994: AVANCE DEL FRENTE DE URBANIZACION EN NANCY. CONSECUENCIAS MORFOLOGICAS Y PAISAJISTICAS}

En las fotografías aéreas de la serie de 1968 se comprueba un avance del frente de urbanización en relación a la superficie edificada de la serie de 1950. Es ahora cuando empieza a difundirse el chalet unifamiliar del tipo Ile-de-France en los municipios orientales de Essey, Malzèville, Seichamps, Pulnoy y Saulxures y en los occidentales de Vandoeuvre, Houdemont y Jarville. En este primer estadio de la periurbanización nanceienne, las nuevas edificaciones son aquellas que corresponden a vastos programas de hábitat individual planificado.

Posteriormente, a mediados de los años setenta, siguen construyéndose numerosas residencias unifamiliares. A partir de este momento, se inicia la construcción de las dos mayores ZAC de la aglomeración, Ludres y Clairlieu (más de mil chalets cada complejo) y se completa la gran zona de lotissements del este de la aglomeración 6 . Aunque sean éstos los enclaves más destacados, cabe señalar que el modelo estereotipado de pavillon aparece en mayor medida en la totalidad de los municipios de la periferia de Nancy. Esta nueva configuración del paisaje, especilizada funcionalmente en la residencia, es lo que llevó en 1.980 a J. Bastié a definir el periurbano de Nancy, al igual que otros del este de Francia, como un periurbanodormitorio.

En la década de los ochenta se ha buscado un equilibrio entre residencia y actividad. Para ello, se han promovido cuatro grandes zonas de actividad económica en la aglomeración: Houdemont-Heillecourt, Ludres, Champ-le-Boeuf y Brabois, que han ido alcanzando un éxito progresivo en esta última década, en lo que se refiere a atracción de capital e implantación de nuevas industrias, en su mayor parte ligeras o de transformación, empresas de alta tecnología, y servicios en general.

De una manera racional en los últimos años, se ha frenado el avance del frente de urbanización, y en numerosos municipios, las nuevas edificaciones tienden a rellenar los vacíos funcionales provocados por el primer movimiento expansivo de corta duración pero de gran intensidad. Este hecho se comprueba bastante bien en municipios como Vandoeuvre, Villers, Houdemont, Ludres, Fléville, Pulnoy, Seichamps o Malzèville.

El paisaje periurbano que ha quedado configurado actualmente en Nancy, muestra un predominio morfológico del chalet tipo Ile-de-France (del que existen a su vez numerosas subtipologías arquitectónicas), agrupado en lotissements o

\footnotetext{
6 Más de 4.000 chalets tiene el conjunto de Seichamps, Pulnoy y Saulxures.
} 
urbanizaciones, el cual responde a un modelo de residencia unifamiliar, planificada y permanentemente habitada, rasgos definitorios de la vivienda periurbana por excelencia. La prefabricación en serie y las facilidades financieras de adquisición han posibilitado que esta tipología resulte asequible a una gran parte de la población.

Son éstas las causas de una democratización del chalet, que en el caso del periurbano de Nancy aparece en la totalidad de los municipios, generando un paisaje monótono y hasta aburrido debido a su planificación conjunta, lo cual tiene como compensación la reducción de los desequilibrios verticales, puesto que existe una planificación y gestión simultánea de infraestructuras y equipamientos, de los que carecería en el caso de haberse producido una periurbanización espontánea, descontrolada y al margen del planeamiento.

Aunque el modelo estereotipado periurbano francés se ejemplifique perfectamente en Nancy, existen sin embargo otras morfologías que complementan y enriquecen el paisaje periurbano en Nancy: el crecimiento difuso en la periferia o los lotissements espontáneos en Tomblaine; los microlotissements o la densificación difusa del núcleo rural en Art-sur-Meurthe; las residencias de lujo integradas en el bosque o cottage en Ludres, Brabois y Bellevue; la convivencia de antiguos y recientes lotissements en Maxèville, Champigneulles, Malzèville; las ciudades obreras de Pompey y Frouard; los grandes complejos de hábitat colectivo en Vandoeuvre, Laxou (Provinces y Champ-le-Boeuf), Tomblaine, Ludres, Heillecourt y St. Max, así como la presencia de los núcleos rurales y la infinidad de tipologías ligadas a una función de ocio, transportes, industrial, comercial o de servicios: campos de deporte de Tomblaine, Ludres, Villers, Heillecourt, Laxou y el Plateau de Malzèville; los nudos de autopista; el centro internacional de transportes de Champigneulle; naves de los polígonos industriales; la tecnópolis de Brabois; las zonas hoteleras de HoudemontHeillecourt y Laxou. De una manera especial se manifiesta en diversos puntos una de las morfologías periurbanas más destacadas, las grandes superficies comerciales.

\section{FUNCIONALIDAD DEL ESPACIO PERIURBANO DE NANCY. OPERACIONES DE AMENAGEMENT}

El estudio del espacio periurbano nanceienne se ha concebido finalmente como la conjunción de medidas específicas de ordenación y aménagement périurbain, las cuales convergen en la individualización y particularización del caso de Nancy. En este sentido, el primer órgano político-administrativo de la aglomeración, el DAN ${ }^{7}$, que

7 Distrito Urbano de Nancy. Agrupa a dieciocho municipios de la aglomeración y tiene plenas competencias en materia de urbanismo previsional y operacional. 
está en constante colaboración con la $\mathrm{ADUAN}^{8}$, ha jugado un rol fundamental en la planificación, gestión y ejecución de una política urbana coherente.

\section{Infraestructuras y transportes}

Como todo espacio periurbano, el de Nancy se halla fuertemente condicionado por el impacto espacial de las autopistas que lo atraviesa. En nuestro caso, existen tres autopistas que discurren por el periurbano: la A-31 (Borgoña-Lorena), la A-33 (ParísEstrasburgo) y la A-330 (Nancy-Epinal), las cuales se entrecruzan en dos grandes nudos, uno al oeste y otro al suroeste.

La congestión de las dos primeras autopistas por un triple uso, europeo, regional y local, ha motivado a estudiar la posibilidad de desdoblar la A-31, construir una variante por fuera de la aglomeración y dejar la actual como eje estructurante periurbano de un modo casi exclusivo. De esta manera se producirá un ahorro de costes y se disminuirá el riesgo de accidentalidad.

La localización de los complejos residenciales y de las zonas de actividad económica, en especial del comercio en tanto que servicio nodal, producen cambios en el territorio y en su organización. La localización periurbana tienen como uno de sus principales factores, la accesibilidad. De ahí que sea clave en la comprensión del periurbano de Nancy, la interacción entre las principales vías de comunicación y los asentamientos más destacados.

Cuando un servicio axil, como una autopista, tiene las salidas bastante distanciadas, éstas se convierten en un servicio nodal y los tramos entre esas salidas son infraestructuras parasitarias para el territorio en el que se asienta. En el periurbano de Nancy, a excepción del tramo que discurre en Laxou por el bosque de hayas, ésto no sucede. El hecho de tener diez salidas en una red periurbana de autopistas inferior a cuarenta kilómetros, supone un fácil acceso a este espacio y una relación directa entre infraestructuras de transporte y asentamientos periurbanos (de residencia o actividad), hasta el punto de que no son infraestructuras parasitarias, sino que se crean en horas y días punta deseconomías por la congestión del tráfico.

Sin embargo existe un desequilibrio horizontal si consideramos la globalidad de la aglomeración. La práctica totalidad de los kilómetros y nueve de esas diez salidas, se encuentran en la margen izquierda de la Meurthe. En la margen derecha el gran eje periurbano es la Nacional 74, con un impacto periurbano menor que las autopistas,

8 Agencia de Desarrollo y de Urbanismo de la Aglomeración de Nancy, principal centro de estudios de la aglomeración, que me ha permitido el acceso a numerosísima documentación urbanística del área de estudio. 
sobre todo en la localización de industrias, debido a que el abastecimiento de materias primas y la salida al mercado de los productos elaborados por medio de grandes camiones se hace de manera más lenta y con mayor dificultad. Con la próxima apertura de la autovía de enlace A-330-Departamental 2-Nacional 74, se espera una mejora de la accesibilidad y una revalorización de este espacio.

El desequilibrio en infraestructuras entre ambas márgenes, ha conducido a que el impacto espacial de la margen derecha esté especializado en la residencia, que no tiene la misma necesidad de comunicaciones, abundantes en la izquierda, de cara a hacer atractiva esta zona para la localización de actividad. El nuevo enlace pretende reducir estos desequilibrios intraperiurbanos y desarrollar de manera complementaria residencia y actividad en los municipios orientales, los cuales, por tener peores infraestructuras de transporte, han tenido un crecimiento periurbano menor.

De manera particular sobresale en el espacio periurbano de Nancy, la construcción de la plataforma logística multimodal de Champigneulles. Es un gran centro de transportes internacional, que aprovecha la coalescencia de tres medios: autopista, ferrocarril y puerto fluvial de la Mosela canalizada. El proyecto apunta la previsión de una superficie global de 150 hectáreas, divididas en dos áreas: un centro de servicios ligados al transporte y a las empresas de distribución, y un complejo de almacenes e industrias de empaquetación, etiquetaje, etc. El presupuesto global de las operaciones se estima en unos 100 millones de francos (2500 millones de pesetas), financiados en su mayor parte por un sindicato mixto que agrupa a las diversas colectividades territoriales. Aunque las obras comenzaron en 1993, la puesta en marcha de la plataforma será una realidad en el año 2000.

\section{Actividad secundaria y servicios}

El rápido avance del frente de urbanización se ha desarrollado sobre las tradicionales grandes parcelas de cultivo de cereal, el típico paisaje de oppenfield de la Lorena. El resultado actual es que sólo ha quedado en las zonas más próximas a Nancy una agricultura-reliquia, que tuvo una expansión mayor en el pasado. De hecho, el porcentaje de población activa dedicada al sector primario en el conjunto de la aglomeración es inferior al $2 \%$.

$\mathrm{Al}$ analizar los polígonos de industria ligera, se observa que éstos suelen estar: complementados en su localización por una diversidad de servicios. De ahí el concepto genérico de Zona de Actividad, que se ha difundido más que el específico de zona industrial. La actividad secundaria aparece en escasas ocasiones individualizada en el espacio periurbano de Nancy. 
Destacan las dos grandes zonas de actividad del sur de la aglomeración, LudresFléville y Houdemont-Heillecourt, más de 250 hectáreas en total. Otras zonas de menor extensión son las de Brabois y St. Jacques. Posteriormente, se percibió la desigualdad territorial respecto a la zona oriental y se ha potenciado la margen derecha de la Meurthe, en especial la ZAC de Pulnoy-Essey.

Puede generalizarse que, como la industria pesada y contaminante se alejó del centro de la aglomeración, se generaron unos espacios industriales periurbanos de transición entre el municipio central y los tres polos de industria de base. Existe por lo tanto, una industria periurbana con una cierta dispersión y diversificación, aunque tal diversidad hay que entenderla de manera relativa. La mayoría de las implantaciones industriales del periurbano de Nancy pueden ser encuadradas dentro del conjunto de industrias ligeras y de transformación: agroalimentarias, transformados metálicos y de la madera, maquinaria, eléctrica, química ligera, imprenta, confección, etc.

Del conjunto de las nuevas áreas de actividad económica puede desprenderse una constante: son zonas que, sin estar contiguas a los grandes conjuntos residenciales, están próximas a ellos. La mayoría de los municipios periurbanos han rechazado una especialización funcional casi exclusiva y ha pretendido una complementariedad entre residencia y actividad. Esta reacción se manifiesta más claramente en los municipios de la margen derecha, donde predominaban las viviendas frente a las zonas de actividad, lo cual concluye en una mayor armonía y equilibrio espacial del propio espacio periurbano de Nancy.

Mención especial merece el nuevo urbanismo comercial de la periferia. Dentro del conjunto de las metrópolis francesas de más de 250.000 habitantes, Nancy ocupa el tercer puesto en densidad de equipamientos comerciales: 464 metros cuadrados de superficie de venta (133 específicamente de hipermercados) por cada 1.000 habitantes. El número de hipermercados se ha incrementado los últimos años, así como la superficie total. Lo que destaca recientemente en el periurbano de Nancy es la. explosión de almacenes de comercio especializado y superficie media (entre $400 \mathrm{y}$ 2.500 metros cuadrados), anexos a los supermercados. La vivienda unifamiliar periurbana genera unas demandas, por ejemplo el arreglo y cuidado del jardín, que son satisfechas en nuevas fórmulas comerciales, tipicamente periurbanas en su localización e identidad.

De los diez hipermercados que existen en la aglomeración, ocho están en la periferia de Nancy. Es interesante conocer su localización por su función de articulación de otras actividades económicas en torno a ellos: restaurantes, superficies medianas de comercio especializado y hoteles (zona de Champ-le-Boeuf y de 
Houdemont-Heillecourt). En general, los ocho hipermercados están situados en las proximidades de las salidas de las autopistas. Estos centros de gran superficie, dinamizan zonas de actividades y servicios, y estructuran y jerarquizan el territorio en función del cono de demanda de los habitantes de los lotissements próximos a ellos y del centro de la aglomeración. El que exista sólo un hipermercado en el municipio central, genera unas economías de aglomeración de las que se benefician los municipios periurbanos.

Dentro de la actividad secundaria, cabe citar el cierre de numerosas plantas siderúrgicas en la Lorena, entre las que se encuentra Pompey, en pleno espacio periurbano de Nancy. La planta de Pompey producía en 1980, 470.000 toneladas anuales de acero fino y tenía 3.700 empleos directos. Después del cierre de la factoría hubo que hacerse cargo de las repercusiones sociales y espaciales. Aparece entonces en la Lorena el EPML ${ }^{9}$, organismo público encargado de frenar la especulación de los solares y de llevar a cabo la comercialización de los terrenos. Desde 1991 se han ido asentando diversas empresas del sector eléctrico en las diferentes ZAC creadas aquí para una optimización en la rehabilitación de los barbechos industriales. Esta política de recuperación de suelo parece tener continuidad con una serie de medidas de ordenación urbana encaminadas a integrar este espacio tan característico con el de la plataforma multimodal de Champigneulles, contigua a los barbechos industriales, dadas las previsibles necesidades de suelo del centro de transportes.

Como conjunto más destacado de operaciones de aménagement périurbain en toda la aglomeración, está la creación del parque tecnológico de Nancy-Brabois. El consenso del Sindicato Intercomunal Nancy-Vandoeuvre (1968) lleva a la creación de la ZAC de Brabois en 1972. A partir de ahí, y gracias a la sociedad de economía mixta Solorem, principal actor en la materialización del proyecto, se realizan los trabajos de urbanización tecnopolitana.

El acondicionamiento y la disponibilidad de terrenos no es suficiente para dinamizar este espacio, de ahí la necesidad de crear un organismo de promoción: Nancy-Brabois-Innovation (NBI), el cual a través de su estrategia de las $4 \mathrm{~A}^{10}$ ha conseguido la implantación progresiva de empresas de alta tecnología y sedes regionales de empresas. Actualmente, las 500 hectáreas del territorio tecnopolitano ${ }^{11}$ no son suficientes y se ha tenido la necesidad de crear dos microparques tecnológicos, en Ludres y Maxèville, para poder acoger a nuevas empresas.

\footnotetext{
${ }^{9}$ Establecimiento Publico de la Metropolis Lorena.

10 Aménagement, Argent, Accueil, Animation.

11 Dentro del que se encuentran ubicados las Facultades de Medicina y Ciencias, las Escuelas Superiores de Ingenieros, así como numerosos laboratorios de investigación adscritos a ellos.
} 
La configuración y crecimiento de la tecnópolis periurbana de Nancy, cuenta con más de una quincena de años. Esto le ha permitido materializar unas estrategias de desarrollo que mejoran la imagen de la aglomeración en el exterior. Al mismo tiempo, el impacto espacial de los diferentes sitios tecnopolitanos le confiere tal riqueza y dinamismo al periurbano de Nancy, que no puede entenderse su ordenación periurbana global sin tener en cuenta el efecto motriz de la tecnópolis.

\section{Función residencial}

Si en la función de actividad y en las infraestructuras existe un cierto desequilibrio entre el este y el oeste, en la función residencial hay un mejor reparto espacial. Aunque el resultado final, morfológico y paisajístico, sea prácticamente el mismo, conviene distinguir los dos procedimientos urbanísticos más empleados en la edificación de un conjunto de chalets: la ZAC y el lotissement. El primero es un programa de mayor número de viviendas, con mayor exigencia en materia de infraestructuras y equipamientos, por lo tanto, con una cierta unidad espacial. El segundo se refiere a procedimientos más sencillos, por lo tanto más numerosos y con una dispersión espacial mayor.

En el caso de Nancy, la ZAC se ha aplicado a las dos mayores urbanizaciones de la aglomeración, Ludres y Clairlieu, dos verdaderas microciudades-jardín, mientras que el lotissement aparece de manera masiva en la margen derecha. Del análisis estadístico de las ZAC y los lotissements que actualmente están en curso de construcción y comercialización, desprendemos que actualmente no se construyen complejos de gran número de chalets, ni muy alejados del centro de la aglomeración. La cierta madurez del proceso de periurbanización exige que no haya más extensión superficial, sino rellenar los huecos funcionales que restan y que se minimicen los desequilibrios. Esto nos hace pensar que el frente de urbanización ralentiza su avance, cuando no es retroactivo.

Pasamos de una situación "del village al lotissement" a otra de "reperiurbanización del propio espacio periurbano". Se llegado a un límite, que por supuesto no es definitivo, en la difusión espacial. Para poder seguir con el crecimiento expansivo se necesita volver atrás para armonizar al territorio metropolitano. 


\section{DINAMISMO E INTERRELACION DEL SISTEMA PERIURBANO DE NANCY. LOS MOVIMIENTOS PENDULARES}

La extrema movilidad es lo que caracteriza al espacio periurbano de Nancy. Nada de lo dicho anteriormente tendría sentido si no se concebiera de manera sistémica. La dinamización e interrelación entre los múltiples espacios periurbanos de Nancy, son definitivos para diseccionar el territorio geográfico global.

Debido a la reducida superficie del municipio central respecto del conjunto del área metropolitana, y debido tambien a la dispersión espacial de las actividades y de las residencias, existe una concluyente movilidad periurbana, superior al $70 \%$ de los activos residentes. El mayor número de intercambios se produce en el interior del propio espacio periurbano y en vehículo individual: 492.000 desplazamientos diarios en 1992, seguido de la circulación entre centro y periferia, tambien en vehículo particular, 134.000 .

La encuesta MIRABELLE expone el porcentaje de población activa que se desplaza diariamente del lugar de residencia al del trabajo. De las 552 interconexiones posibles, puede generalizarse lo expuesto anteriormente: hay una interrelación de "todos con todos" a excepción de los municipios de la margen derecha.

Los municipios con mayor porcentaje de activos que salen a trabajar a otro, son aquellos que albergan las grandes áreas residenciales, Fléville (89\%), Villers, Seichamps, Pulnoy, Saulxures y Bouxières, y viceversa, los municipios con mayor porcentaje de entradas son los de las grandes zonas de actividad, Ludres, Houdemont y Heillecourt.

La conclusión que desprendemos de la movilidad diaria es, que el espacio periurbano de Nancy es abundante en desplazamientos habituales, lo cual le concede un carácter bastante dinámico. Ahora bien, estos desplazamientos siguen unos flujos muy concretos entre las zonas de actividad y residencia de la aglomeración, produciéndose una congestión del tráfico periurbano en determinados momentos del día. La dispersión espacial de ambos y la mejora en las infraestructuras es lo que debe conducir a una mejor articulación del espacio periurbano de Nancy en un futuro no muy lejano. 


\section{CONCLUSIONES: LA SEMANTICA PERIURBANA DE NANCY}

El sistema periurbano de Nancy aparece finalmente como un sistema intraurbano en el que los 24 municipios tienen una relación entre ellos mismos, con el municipio central y con el resto del territorio regional, francés y europeo.

Se define por un paisaje contrastado y diversificado. Aunque el protagonismo del hábitat individual en la morfología es manifiesto, sin embargo se complementa con otras formas de construcción. De esta manera, se configura un paisaje heterogéneo, en el que hay una tipología predominante.

La plurifuncionalidad de los usos del suelo es otra expresión del significado periurbano en Nancy. La convivencia entre espacios residenciales, agrarios, industriales, comerciales, y de servicios, ha exigido una planificación y ordenación urbana con el fin de evitar incompatibilidades en los usos del mismo.

Este análisis periurbano en Nancy nos puede dar las claves de la política metropolitana. Creo que son tres, los grandes retos de la ordenación urbana de la aglomeración:

- Delimitar el crecimiento expansivo de la aglomeración

- Reducir los desequilibrios horizontales entre ambas márgenes de la Meurthe

- Profundizar la tendencia de complementar residencia y actividad, con una adecuada localización y aplicación de las ZAC.

Finalmente, la síntesis de la disparidad en las formas y en las funciones, converge paradójicamente en la unidad de significado periurbano en Nancy, en tanto en cuanto este espacio actúa de manera global consigo mismo y en sus relaciones con el municipio central, bien sea por la conciencia que de ello tienen sus habitantes, bien por la preocupación integral de sus responsables políticos y urbanísticos, que se ocupan de minimizar los desequilibrios verticales y horizontales, concluyendo así por ofrecer un espacio bastante jerarquizado, estructurado y armónico en su conjunto.

\section{BIBLIOGRAFIA}

ADUAN (1993): Atlas de l'agglomeration nanceienne. Nancy.

BAUER, G. et ROUX, J. (1976): La périurbanisation ou la ville éparpiléé. Seuil. París. 
BRUNET, P. (1982): Carte des mutations de l'espace rural français.1950-1980.CNRS.Caen.

Cahiers du CREPIF n 3 . (1983): Peut-on maîtriser le développement périurbain? París.

DEZERT, B., METTON, A. y STEINBERG, J. (1991): La périurbanisation en France. SEDES. París.

DION, R. (1983): La région urbaine de Nancy. Tesis Doctoral. Université de Nancy II. 